\title{
ON SOME PRODUCTS OF $\beta$-ELEMENTS IN THE STABLE HOMOTOPY OF $L_{2}$-LOCAL SPHERES
}

\author{
Reiko Otsubo and Katsumi Shimomura
}

\section{$\S 1$. Introduction}

The $\beta$-elements in the stable homotopy groups of spheres at the prime $>3$ are introduced by H. Toda ([22]) and generalized by L. Smith ([21]) and S. Oka $([4],[5],[6])$. In [3], H. Miller, D. Ravenel and S. Wilson give the way to define the generalized Greek letter elements, including $\beta$-elements, in the $E_{2^{-}}$ term of the Adams-Novikov spectral sequence for computing the homotopy groups $\pi_{*}\left(S^{0}\right)$. S. Oka ([7], [8]) and H. Sadofsky ([12]) show that some of them are permanent cycles in the spectral sequence.

The second author has studied about the product of these $\beta$-elements $([9]$, $[13],[14],[15],[16],[17])$. The $\beta$-elements of the homotopy groups $\pi_{*}\left(M_{p}\right)$ of the mod $p$ Moore spectrum $M_{p}$ appear when we define those of $\pi_{*}\left(S^{0}\right)$. In fact, a $\beta$-element $\beta_{t}^{\prime}$ of $\pi_{*}\left(M_{p}\right)$ is sent to $\beta_{t}$ in $\pi_{*}\left(S^{0}\right)$ by the projection map $\pi: M_{p}$ $\rightarrow \Sigma^{1} S^{0}$ to the top cell. It is also studied the non-triviality of products $\beta_{t}^{\prime} \beta_{E}$ of $\beta$-elements $\beta_{t}^{\prime}$ in $\pi_{*}\left(M_{p}\right)$ and $\beta_{E}$ in $\pi_{*}\left(S^{0}\right)$ for some subscript $E$ (cf. [18], [1], [2]). In this paper, we study the projection map $\pi: M_{p} \rightarrow \Sigma^{1} S^{0}$, and try to push out the non-trivial products of the homotopy groups of the Moore spectrum $M_{p}$ to those of the sphere spectrum $S^{0}$. In other words, we study whether $\beta_{t} \beta_{E}$ is nontrivial in $\pi_{*}\left(S^{0}\right)$ when $\beta_{t}^{\prime} \beta_{E}$ is non-trivial.

By the recent work [20], A. Yabe and the second author have determined the additive structure of the homotopy groups of $L_{2}$-local spheres, where $L_{2}$ stands for the Bousfield localization functor with respect to the Johnson-Wilson spectrum $E(2)$ whose coefficient ring is $\boldsymbol{Z} / p\left[v_{1}, v_{2}, v_{2}^{-1}\right]$. Then we have the localization map $\pi_{*}\left(S^{0}\right) \rightarrow \pi_{*}\left(L_{2} S^{0}\right)$. It would be fine if we obtain some information of $\pi_{*}\left(S^{0}\right)$ from the map, but we do not treat it here. Actually we study, in this paper, the localized map $L_{2} \pi: L_{2} M_{p} \rightarrow L_{2} \Sigma^{1} S^{0}$ rather than $\pi$ itself.

In particular, in [2] and [1], we have shown a relation

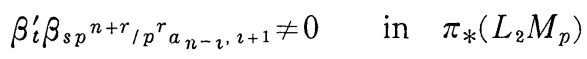

under the following condition on the integers appeared in the subscripts of $\beta$ 's.

$$
p \nmid \text { st for even } r \geqq 2 \text {, and }
$$

Received February 21, 1994 ; revised June 13, 1994. 
$p \mid c$ and $p \nmid c+p$ for odd $r \geqq 1$.

Here $a_{\imath}$ denotes the integer $p^{\imath}+p^{\imath-1}-1$ if $i>0$ and 1 if $i=0$, and $c$ is an integer such that

$$
t+s p^{n+r}-p^{n+r-\imath-1}+\left(p^{r}+1\right) /(p+1)=c p^{l}-\left(p^{l}-1\right) /(p-1) \text { and } p \nmid c+1
$$

for some $l \geqq 0$. Note that the definition of $\beta$-elements is slightly different from that of [3]. For our elements, see $\S 2$. Further note that $\beta_{s p^{n+r} / p^{r} a_{n-\imath, \imath+1}}$ is defined if $0<i+1 \leqq r$ and $i \leqq n$. Our main result is that the above products of $\beta$ 's in $\pi_{*}\left(L_{2} M_{p}\right)$ all survive to $\pi_{*}\left(L_{2} S^{0}\right)$ under the map $L_{2} \pi_{*}$, and so we have

THEOREM. Let $t, s, n, r$ and $i$ be non-negative integers such that $t, s, r>0$ and $i \leqq \min \{r-1, n\}$. In the homotopy groups $\pi_{*}\left(L_{2} S^{0}\right)$, the product $\beta_{t} \beta_{s p^{n+r}}$, $p^{r} a_{n-\imath, \imath+1}$ is not null if the condition (1.1) is satisfied.

As an example, taking $r=1$, we have

COROLlary. Let $u, s$ and $n$ be positive integers. Then,

if $n>1$, and

$$
\beta_{u p^{2}-1} \beta_{s p^{n+1} / p^{n+1}+p^{n}-p} \neq 0 \in \pi_{*}\left(L_{2} S^{0}\right)
$$

if $n>2$.

$$
\beta_{u p^{3}-2} \beta_{s p^{n+1} / p^{n+1}+p^{n}-p} \neq 0 \in \pi_{*}\left(L_{2} S^{0}\right)
$$

\section{$\S 2$. $\beta$-elements}

Let $(A, \Gamma)$ denote the Hopf algebroid associated to the Johnson-Wilson spectrum $E(2)$ with coefficient ring $E(2)_{*}=Z_{(p)}\left[v_{1}, v_{2}, v_{2}^{-1}\right]$ :

$$
A=E(2)_{*} \quad \Gamma=E(2)_{*}(E(2))=E(2)_{*}\left[t_{1}, t_{2}, \cdots\right] \otimes_{B P_{*}} E(2)_{*},
$$

in which $B P_{*}$ acts on $E(2)_{*}$ by sending $v_{n}$ to $v_{n}$ if $n \leqq 2$, and to 0 if $n>2$. Then there is the Adams-Novikov spectral sequence converging to $\pi_{*}\left(L_{2} S_{0}\right)$ $\left(\right.$ resp. $\left.\pi_{*}\left(L_{2} M_{p}\right)\right)$ with $E_{2}$-term $E_{2}^{*}=\operatorname{Ext}_{\Gamma}^{*}(A, A)\left(\operatorname{resp.} E_{2}^{*}=\operatorname{Ext}_{\Gamma}^{*}(A, A /(p))\right)$. Here in this paper, an element of the Ext-groups will be represented by an element of the cobar complex $\Omega_{\Gamma}^{*} A$ (resp. $\left.\Omega_{\Gamma}^{*} A /(p)\right)$. We shall abbreviate $\operatorname{Ext}_{\Gamma}^{s}(A, M)$ by

\section{$\operatorname{Ext}^{s}(M)$}

for a $\Gamma$-comodule $M$. We see that $E_{2}^{s}=0$ for $s>4$ by using Morava's theorem [10] (cf. [3, Th. 3.6], [11, Ch. 6]) and the chromatic spectral sequence [3, 3.A] (cf. [11, Ch. 5]). Therefore the spectral sequence collapses and arises no extension problem by its sparseness. Hence we identify the $E_{2}$-term with its abutment $\pi_{*}\left(L_{2} S^{0}\right)$ or $\pi_{*}\left(L_{2} M_{p}\right)$.

In order to define the $\beta$-elements, consider the connecting homomorphisms 


$$
\begin{aligned}
& \delta_{1}: \operatorname{Ext}^{1}\left(A /\left(p^{\imath+1}\right)\right) \longrightarrow \operatorname{Ext}^{2}(A), \text { and } \\
& \delta_{0}: \operatorname{Ext}^{0}\left(A /\left(p^{\imath+1}, v_{1}^{\jmath}\right)\right) \longrightarrow \operatorname{Ext}^{1}\left(A /\left(p^{\imath+1}\right)\right)
\end{aligned}
$$

associated to the short exact sequences

$$
\begin{gathered}
0 \longrightarrow A \stackrel{p^{2+1}}{\longrightarrow} A \longrightarrow A /\left(p^{2+1}\right) \longrightarrow 0 \text { and } \\
0 \longrightarrow A /\left(p^{2+1}\right) \stackrel{v_{1}^{\jmath}}{\longrightarrow} A /\left(p^{\imath+1}\right) \longrightarrow A /\left(p^{2+1}, v_{1}^{\jmath}\right) \longrightarrow 0,
\end{gathered}
$$

respectively. Here we assume that

$$
p^{\imath} \mid j
$$

In [9], Miller, Ravenel and Wilson introduced the elements $x_{n} \in v_{2}^{-1} B P_{*}$ defined by

$$
\begin{aligned}
& x_{0}=v_{2}, \\
& x_{1}=v_{2}^{p}-v_{1}^{p} v_{2}^{-1} v_{3} \\
& x_{2}=x_{1}^{p}-v_{1}^{p^{2}-1} v_{2}^{p^{2}-p+1}-v_{1}^{p^{2}+p-1} v_{2}^{p^{2}-2 p} v_{3} \\
& x_{n}=x_{n-1}^{p}-2 v_{1}^{a} n^{n-p} v_{2}^{p^{n}-p^{n-1}+1} \quad \text { for } \quad v \geqq 3,
\end{aligned}
$$

where $a_{n}=p^{n}+p^{n-1}-1$ for $n>0$ and $a_{0}=1$, and showed that

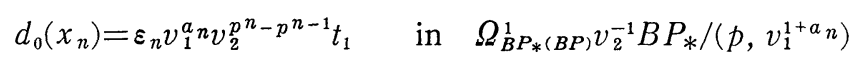

for $n>0$ and $\varepsilon_{n}=\min \{n, 2\}$. Here

$$
d_{0}=\eta_{R}-\eta_{L}: v_{2}^{-1} B P_{*} \longrightarrow \Omega_{B P_{*}(B P)}^{1} v_{2}^{-1} B P_{*} /\left(p, v_{1}^{1+a_{n}}\right)
$$

for the right and the left units $\eta_{R}$ and $\eta_{L}$ of the Hopf algebroid $B P_{*}(B P)$. Note that $x_{n}^{s}$ belongs to $B P_{*} /\left(p^{2+1}, v_{1}^{j}\right)$ if $p^{\imath} \mid j \leqq a_{n-\imath}(c f .[3])$. In other words, if $p^{\imath} \mid j \leqq a_{n-\imath}, x_{n}^{s} \in v_{2}^{-1} B P_{*} /\left(p^{\imath+1}, v_{1}^{j}\right)$ is pulled back to $B P_{*} /\left(p^{\imath+1}, v_{1}^{j}\right)$ under the localization map $B P_{*} \subseteq v_{2}^{-1} B P_{*}$. Thus we may consider that $x_{n}^{s}$ is in $B P_{*} /\left(p^{2+1}\right.$, $\left.v_{1}^{\jmath}\right)$ not in $v_{2}^{-1} B P_{*}$, and (2.3) shows

$$
x_{n}^{s} \in \operatorname{Ext}_{B P_{*}(B P)}^{0}\left(B P_{*}, B P_{*} /\left(p^{\imath+1}, v_{1}^{\jmath}\right)\right) \subset B P_{*} /\left(p^{\imath+1}, v_{1}^{\jmath}\right)
$$

under the condition, which yields the $\beta$-element $\beta_{s p n / \jmath, \imath+1}$ as the image under the composition of the connecting homomorphisms associated to the short exact sequences

$$
\begin{gathered}
0 \longrightarrow B P_{*} \stackrel{p^{\imath+1}}{\longrightarrow} B P_{*} \longrightarrow B P_{*} /\left(p^{\imath+1}\right) \longrightarrow 0 \text { and } \\
0 \longrightarrow B P_{*} /\left(p^{\imath+1}\right) \stackrel{v_{1}^{\jmath}}{\longrightarrow} B P_{*} /\left(p^{\imath+1}\right) \longrightarrow B P_{*} /\left(p^{\imath+1}, v_{1}^{\jmath}\right) \longrightarrow 0 .
\end{gathered}
$$

Considering this condition we have 
(2.4) $\left[3\right.$, Th. 2.6] Let $E_{2}^{s, t}=\operatorname{Ext}_{B P_{*}(B P)}^{s, t}\left(B P_{*}, B P_{*}\right)$ denote the $E_{2}$-term of the Adams-Novikov spectral sequence for $\pi_{*}\left(S^{0}\right)$. Then $E_{2}^{2, *}$ consists of the $\beta$ elements $\beta_{s p n / \jmath, 2+1}$ with

$$
p \nmid s, \quad p^{2} \mid j \leqq a_{n-\imath} \text { and } j \leqq p^{n-\imath} \text { if } s=1 .
$$

In the following, we define the $\beta$-elements in the $E_{2}$-term $\operatorname{Ext*}(A)$ of the Adams-Novikov spectral sequence computing $\pi_{*}\left(L_{2} S^{0}\right)$. As we have noted above, these $\beta$-elements are considered to be homotopy elements. Then $\beta$-elements in the $\pi_{*}\left(S^{0}\right)$ are obtained by pulling back those elements under the localization map $\eta: S^{0} \rightarrow L_{2} S^{0}$.

Consider the map $f: v_{2}^{-1} B P_{*} \rightarrow A$ given by sending $v_{n}$ to $v_{n}$ if $n \leqq 2$ and to 0 otherwise. We define the elements $x_{n}$ in $A$ by sending those in $v_{2}^{-1} B P_{*}$ to $A$ under the map $f$. Actually they are obtained by setting $v_{3}=0$, and yield the same results (2.3). This with (2.3) implies that

$$
x_{n}^{s} \in \operatorname{Ext}^{0}\left(A /\left(p^{\imath+1}, v_{1}^{j}\right)\right) \quad \text { for } \quad p^{\imath} \mid j \leqq a_{n-\imath},
$$

and further that

$$
x_{n-\imath}^{s p r+\imath} \in \operatorname{Ext}^{0}\left(A /\left(p^{\imath+1}, v_{1}^{j}\right)\right) \quad \text { for } \quad p^{\imath} \mid j \leqq a_{n+r-\imath} .
$$

Using these elements, we define the $\beta$-elements by

$$
\begin{aligned}
& \beta_{s p n+r / \jmath}^{\prime}=\delta_{0}\left(x_{n-2}^{s p^{r+2}}\right) \in \operatorname{Ext}^{2}(A /(p)) \quad \text { for } 0<j \leqq a_{n} \\
& \beta_{s p n+r / \jmath, \imath+1}=\delta_{1} \delta_{0}\left(x_{n-\imath}^{s p^{r+2}}\right) \in \operatorname{Ext}^{2}(A) \\
& \quad \text { for } \quad p^{\imath} \mid \jmath \text { with } p^{r+1} a_{n-\imath-1}<j \leqq p^{r} a_{n-\imath}
\end{aligned}
$$

in the $E_{2}$-terms of the Adams-Novikov spectral sequences computing $\pi_{*}\left(M_{p}\right)$ and $\pi_{*}\left(S^{0}\right)$. Here we notice that $\beta$-elements in [3] are defined by using $x_{n}$ instead of $x_{n-\imath}^{p^{2}}$ as we have done here. The subscripts of $\beta$-elements are given as follows:

$$
\beta_{a / b, c}=\delta_{1} \delta_{0}\left(v_{2}^{a}+v_{1} x\right)
$$

for some $x \in B P_{*}$ such that

$$
v_{2}^{a}+v_{1} x \in \operatorname{Ext}^{0}\left(A /\left(p^{c}, v_{1}^{b}\right)\right) .
$$

Thus our $\beta$ 's are good to be considered. We abbreviate $\beta_{s p n / \jmath, 1}$ to $\beta_{s p n / \jmath}$, $\beta_{s p n / 1}$ to $\beta_{s p n}$ and $\beta_{s p n / 1}^{\prime}$ to $\beta_{s p n}^{\prime}$ as is our custom.

We end this section by stating the following.

LEMMA 2.6. ([1, Lemma 3.8]) Let $s, n, r, j$ and $i$ be integers such that $p \nmid s>0, r>0, n>i \geqq 0, p^{\imath} \mid \jmath, 1 \leqq \jmath \leqq p^{r} a_{n-\imath}$ and $r \geqq i$. Then in $\operatorname{Ext}^{2}(A)$, we have 


$$
\beta_{s p n+r / \jmath, \imath+1} \equiv\left\{\begin{array}{c}
-\varepsilon_{n-i} s v_{1}^{p r a} a_{n-i-\jmath} v_{2}^{e(s, n+r ; \imath, r)} g_{0} \bmod \left(p, v_{1}^{p r a_{n-i-\jmath+1}}\right) \\
\text { for even } r, \text { and } \\
-\varepsilon_{n-i} s v_{1}^{p r} a_{n-i-\jmath} v_{2}^{e(s, n+r ; \imath, r)} g_{1} \bmod \left(p, v_{1}^{\left.p^{r} a_{n-i-\jmath+1}\right)}\right. \\
\text { for odd } r .
\end{array}\right.
$$

Here $g_{0}$ and $g_{1}$ are cocycles (cf. [18]) of the cobar complex $\Omega_{\Gamma} A /\left(p, v_{1}\right)$ as follows:

$$
g_{0}=v_{2}^{-p}\left(t_{1} \otimes t_{2}^{p}+t_{2} \otimes t_{1}^{p^{2}}\right) \quad \text { and } \quad g_{1}=v_{2}^{-1} g_{0}^{p} \text {, }
$$

and the integers are defined by:

$$
\begin{gathered}
\varepsilon_{n}=\min \{2, n\}, \quad a_{n}=p^{n}+p^{n-1}-1 \quad \text { and } \\
e(s, n ; i, r)=s p^{n}-p^{n-\imath-1}+k(r),
\end{gathered}
$$

for $k(r)=\left(p^{n}-(-1)^{n}\right) /(p+1)$.

\section{§ 3. The map $L_{2} S^{0} \rightarrow L_{2} M_{p}$}

Consider the cofibering $S^{0} \stackrel{p}{\rightarrow} S^{0} \stackrel{\imath}{\rightarrow} M_{p} \stackrel{\pi}{\rightarrow} \Sigma^{1} S^{0}$ defining the mod $p$ Moore spectrum. Then by [11, Th. 2.3.4] the map $\pi$ induces the map of $E_{2}$-terms

$$
\delta: \operatorname{Ext}^{s}(A / p) \longrightarrow \operatorname{Ext}^{s+1}(A)
$$

By definition we have

$$
\delta\left(\beta_{t}^{\prime}\right)=\beta_{t}
$$

To study this, we consider $\Gamma$-comodules $N_{\jmath}^{\imath}$ and $M_{\jmath}^{\imath}$ introduced in [3]. These are characterized inductively by $N_{0}^{0}=A, N_{1}^{0}=A /(p), M_{\jmath}^{\imath}=v_{\imath+j}^{-1} N_{\jmath}^{\imath}$ and the short exact sequences

$$
0 \longrightarrow N_{\jmath}^{\imath} \longrightarrow M_{\jmath}^{\imath} \longrightarrow N_{\jmath}^{\imath+1} \longrightarrow 0
$$

Note that $M_{\jmath}^{\imath}=N_{\jmath}^{\imath}$ if $i+j=2$. Then by a result of [3], we see that the connecting homomorphisms yield isomorphisms

$$
\operatorname{Ext}^{2}\left(M_{1}^{1}\right) \stackrel{\delta_{1}}{\cong} \operatorname{Ext}^{3}(A /(p)) \text { and } \operatorname{Ext}^{2}\left(M_{0}^{2}\right) \stackrel{\delta_{0}^{\prime}}{\cong} \operatorname{Ext}^{3}\left(M_{0}^{1}\right) \stackrel{\delta_{0}}{\cong} \operatorname{Ext}^{4}(A) \text {. }
$$

In fact, the first isomorphism follows from the fact $\operatorname{Ext}^{s}\left(M_{1}^{0}\right)=0$ for $s>1([3$, Th. 3.16]), the second follows from $\operatorname{Ext}^{s}\left(M_{0}^{1}\right)=0$ for $s>1$ ([3, Th. 4.2]), and the third from $\operatorname{Ext}^{s}\left(M_{0}^{0}\right)=0$ for $s>0$ ([3, Th. 3.16]). Furthermore, note that the isomorphism $\operatorname{Ext}^{2}\left(M_{0}^{2}\right) \cong \operatorname{Ext}^{3}\left(M_{0}^{1}\right)$ is valid at the internal degree $\neq 0$ by $[3$, Th. 4.2]. By definition, we have a canonical inclusions $\varphi: N_{1}^{\imath} \rightarrow N_{0}^{\imath+1}$ and $\varphi$ : $M_{1}^{\imath \rightarrow} M_{0}^{2+1}$ given by $\varphi(x)=x / p$ in both cases. This gives rise to the commutative diagram 


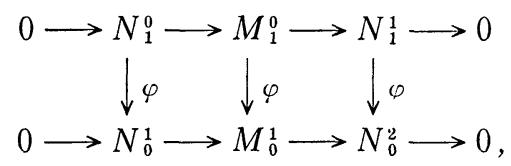

in which two rows are the short exact sequences of (3.3). This diagram yields the commutative one

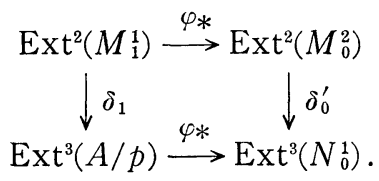

Here note that $N_{1}^{0}=A / p, N_{1}^{1}=M_{1}^{1}$ and $N_{0}^{2}=M_{0}^{2}$. Therefore, the map $\delta$ of (3.1) is identified with

$$
\varphi_{*}: \operatorname{Ext}^{2}\left(M_{1}^{1}\right) \longrightarrow \operatorname{Ext}^{2}\left(M_{0}^{2}\right) .
$$

In fact, $\delta=\delta_{0} \varphi_{*}=\delta_{0} \delta_{0}^{\prime} \varphi_{*} \delta_{1}^{-1}$, and $\delta_{0} \delta_{0}^{\prime}$ and $\delta_{1}$ are the isomorphisms in (3.4). We also have a short exact sequence

$$
0 \longrightarrow M_{1}^{1} \stackrel{\varphi}{\longrightarrow} M_{0}^{2} \stackrel{p}{\longrightarrow} M_{0}^{2} \longrightarrow 0,
$$

which induces the exact sequence

$$
\operatorname{Ext}^{1}\left(M_{0}^{2}\right) \stackrel{\delta}{\longrightarrow} \operatorname{Ext}^{2}\left(M_{1}^{1}\right) \stackrel{\varphi *}{\longrightarrow} \operatorname{Ext}^{2}\left(M_{0}^{2}\right)
$$

Thus we have

LEMMA 3.7. The kernel of $\delta$ in (3.1) is isomorphic to the image of $\delta$ in (3.6).

\section{$\S 4$. Proof of Theorem}

As in Lemma 2.6, we have the cocycles $g_{0}$ and $g_{1}$ representing the generators of $\operatorname{Ext}^{2}\left(M_{2}^{0}\right)$ given by

$$
g_{0}=v_{2}^{-p}\left(t_{1} \otimes t_{2}^{p}+t_{2} \otimes t_{1}^{p^{2}}\right) \text { and } g_{1}=v_{2}^{-1} g_{0}^{p} .
$$

Then in [13], it is shown that $\operatorname{Ext}^{2}\left(M_{1}^{1}\right)$ contains $F_{p}\left[v_{1}\right]$-module

$$
\begin{gathered}
G=F_{p}\left[v_{1}\right]\left\{v_{2}^{s p^{n}-\left(p^{n-1}-1\right) /(p-1)} g_{1} / v_{1}^{a} n \mid n \geqq 1, p \nmid s+1\right\} \\
\oplus F_{p}\left\{v_{2}^{s} g_{0} / v_{1} \mid p \nmid s+1\right\} .
\end{gathered}
$$

Here $a_{0}=1$ and $a_{n}=p^{n}+p^{n-1}-1(n>0)$. In $[20, \S 9]$, the $F_{p}$-module $G_{C}=$ $G /((\operatorname{Im} \delta) \cap G)$ is given by 


$$
\begin{aligned}
G_{C}= & F_{p}\left\{v_{2}^{s p^{n}-\left(p^{n-1}-1\right) /(p-1)} g_{1} / v_{1}^{3} \mid n \geqq 1, p \nmid s+1\right. \\
& 1 \leqq j \leqq a_{n}, p^{\imath+1} \nmid \jmath+A_{n-\imath+1}+1 \text { for } s=u p^{2} \text { with } p \nmid u(u+1), \text { or } \\
& \left.p^{\imath} \nmid j+A_{n-i}+1 \text { for } s=u p^{\imath} \text { with } i>0 \text { and } p^{2} \mid u+1\right\} \\
& \oplus F_{p}\left\{v_{2}^{s} g_{0} / v_{1} \mid p \nmid s+1\right\} .
\end{aligned}
$$

Here

$$
A_{n}=(p+1)\left(p^{n}-1\right) /(p-1) .
$$

LEMMA 4.1. Let $a, b$ and $t$ be positive integers.

1) Put $\beta \equiv v_{1}^{a} v_{2}^{b} g_{0} \bmod \left(p, v_{1}^{a+1}\right)$ in the cobar complex $\Omega_{\Gamma} A$. Then,

$$
\beta_{t} \beta \neq 0
$$

if $a=1$ and $p \nmid t+b+1$.

2) Put $\beta \equiv v_{1}^{a} v_{2}^{b} g_{1} \bmod \left(p, v_{1}^{a+1}\right)$ in the cobar complex $\Omega_{\Gamma} A$. Then,

$$
\beta, \beta \neq 0
$$

if $a=1$ and $p \mid c$ and $p^{2} \nmid c+p$, where $t+b=c p^{l}-\left(p^{l}-1\right) /(p-1)$ with $p \nmid c+1$ for some $l \geqq 0$.

Proof. In the proof of [1, Lemma 4.4], we have seen that $v_{2}^{t} \beta / v_{1}$ is not zero in $\operatorname{Ext}^{2}\left(M_{1}^{1}\right)$ if the conditions of 1$)$ or 2) is satisfied. By the assumption, $v_{2}^{t} \beta / v_{1}$ belongs to $G$ and if it satisfies the conditions of 1 ) or 2), it belongs to $G_{C}$. By Lemma 3.7, $G_{C}$ maps to $\operatorname{Ext}^{2}\left(M_{0}^{2}\right)$ monomorphically. Thus, noticing that $\beta_{t} \beta=\delta_{0} \delta_{0}^{\prime}\left(v_{2}^{t} \beta / v_{1}\right)$, we have the non-trivial products. q.e.d.

Proof of Theorem. By Lemma 2.6,

$$
\beta_{s p n+r / p r a_{n-r, \imath+1}}=-\varepsilon_{n-i} s v_{2}^{s p^{n}-p^{n-\imath-1+k(r)}} g_{\varepsilon(r)}
$$

for $\varepsilon(r)=\left(1-(-1)^{r}\right) / 2$. Now apply Lemma 4.1, and we have Theorem. q.e.d.

\section{REFERENCES}

[1] H. Inoue, Y. Osakada and K. Shimomura, On some products of $\beta$-elements in the homotopy of the Moore spectrum II, to appear in Math. J. Okayama Univ.

[2] M. Mabuchi and K. Shimomura, On some products of $\beta$-elements in the homotopy of the Moore spectrum, Math. J. Okayama Univ., 34 (1992), 195-204.

[3] H. R. Miller, D.C. Ravenel and W.S. Wilson, Periodic phenomena in the Adams-Novikov spectral sequence, Ann. of Math., 106 (1977), 469-516.

[4] S. OKA, A new family in the stable homotopy groups of spheres, Hiroshima Math. J. 5 (1975), 87-114.

[5] S. OKA, A new family in the stable homotopy groups of sheres II, Hiroshima Math. J. 6 (1976), 331-342.

[6] S. ОкА, Realizing some cyclic $B P_{*}$ modules and applications to stable homotopy of spheres, Hiroshima Math. J., 7 (1977), 427-447. 
[7] S. OKA, Small ring spectra and p-rank of the stable homotopy of spheres, Proceedings of the Northwestern Homotopy Theory Conference, Contemp. Math., 19, Amer. Math. Soc., 1983, 267-308.

[8] S. OKA, Multiplicative structure of finite ring spectra and stable homotopy of spheres, Proceedings of the Aahus Algebraic Topology Conference 1982, Lecture Notes in Math., 1051, Springer, 1984, 418-441.

[9] S. OKa AND K. Shimomura, On products of the $\beta$-elements in the stable homotopy groups of spheres, Hiroshima Math. J., 12 (1982), 611-626.

[10] D. C. Ravenel, The cohomology of the Morava stabilizer algebra, Math. Z., 152 (1977), 287-297.

[11] D. C. Ravenel, Complex Cobordism and Stable Homotopy Groups of Spheres, Academic Press, 1986.

[12] H. Sadofsky, Higher p-torsion in the $\beta$-family, Proc. Amer. Math. Soc., 108 (1990), 1063-1071.

[13] K. Shimomura, On the Adams-Novikov spectral sequence and products of $\beta$ elements, Hiroshima Math. J., 16 (1986), 209-224.

[14] K. Shimomura, Non-triviality of some products of $\beta$-elements in the stable homotopy of spheres, Hiroshima Math. J., 17 (1987), 349-353.

[15] K. Shimomura, Triviality of products of $\beta$-elements in the stable homotopy group of spheres, J. Math. Kyoto Univ., 29 (1989), 57-67.

[16] K. Shimomura, On the products $\beta_{s} \beta_{t}$ in the stable homotopy groups of spheres, Hiroshima Math. J., 19 (1989), 347-354.

[17] K. Shimomura, The products $\beta_{s} \beta_{t p / p}$ in the stable homotopy of $L_{2}$-localized spheres, to appear in Hiroshima Math. J.

[18] K. Shimomura and H. TAmura, Non-triviality of some compositions of $\beta$ elements in the stable homotopy of the Moore spaces, Hiroshima Math. J., 16 (1986), 121-133.

[19] K. Shimomura And A. Yabe, On the chromatic $E_{1}$-term $H^{*} M_{0}{ }^{2}$, Topology and Representation Theory, Friedlander and Mahowald ed., Contemp. Math., 158, Amer. Math. Soc., 1994, 217-228.

[20] K. Shimomura And A. YaBe, The homotopy groups $\pi_{*}\left(L_{2} S^{0}\right)$, Topology, 34 (1995), 261-289.

[21] L. Smith, On realizing complex bordism modules, IV, Applications to the stable homotopy groups of spheres, Amer. J. Math., 99 (1971), 418-436.

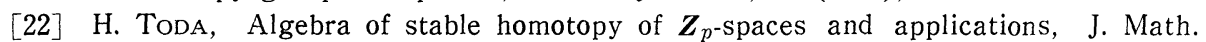
Kyoto Univ., 11 (1971), 197-251.

Department of Mathematics

FACULTy OF EduCATION

TOTTORI UNIVERSITY 